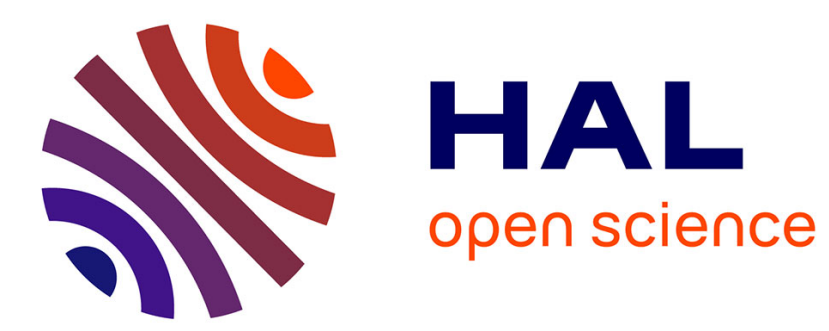

\title{
Retrait vertical d'un sol argileux au cours du dessèchement. Mesures de l'affaissement et conséquences structurales \\ Vincent Hallaire
}

\section{- To cite this version:}

Vincent Hallaire. Retrait vertical d'un sol argileux au cours du dessèchement. Mesures de l'affaissement et conséquences structurales. Agronomie, 1987, 7 (8), pp.631-637. hal-00885036

\section{HAL Id: hal-00885036 https://hal.science/hal-00885036}

Submitted on 1 Jan 1987

HAL is a multi-disciplinary open access archive for the deposit and dissemination of scientific research documents, whether they are published or not. The documents may come from teaching and research institutions in France or abroad, or from public or private research centers.
L'archive ouverte pluridisciplinaire HAL, est destinée au dépôt et à la diffusion de documents scientifiques de niveau recherche, publiés ou non, émanant des établissements d'enseignement et de recherche français ou étrangers, des laboratoires publics ou privés. 


\title{
Retrait vertical d'un sol argileux au cours du des- sèchement. Mesures de l'affaissement et consé- quences structurales
}

\author{
Vincent HALLAIRE (*) \\ I.N.R.A. Station de Science du Sol. Centre de Recherches d'Avignon. F 84140 Montfavet
}

RÉSUMÉ

\begin{abstract}
Le retrait vertical d'un sol riche en argile gonflante est étudié à deux échelles : sur des agrégats naturels d'environ $1 \mathrm{~cm}$ de diamètre, et, in situ, sur des couches de sol d'épaisseur initiale $20 \mathrm{~cm}$. La diminution d'épaisseur due au retrait vertical, ou affaissement du sol, est reliée à son humidité. Ces données expérimentales sont confrontées aux valeurs théoriques d'un affaissement calculé selon l'hypothèse d'un retrait isotrope des agrégats, et de leur empilement continu au sein d'une couche. L'isotropie du retrait est mise en évidence au niveau de l'agrégat. L'affaissement des couches de sol s'opère en 2 temps que l'on explique par une modification de l'organisation structurale : les éléments structuraux s'écartent au début du dessèchement, puis se rapprochent dans une deuxième phase du dessèchement.
\end{abstract}

Mots clés additionnels : Retrait du sol, densité apparente, structure du sol, porosité structurale.

Vertical shrinkage of a clayey soil during drying. Measurements of the sinking and structural consequences.

The vertical shrinkage of a swelling clayey soil was studied at two scales : on natural aggregates of about $1 \mathrm{~cm}$ diameter, and, in situ, on soil layers of $20 \mathrm{~cm}$ initial thickness. The thickness decrease due to vertical shrinkage, i.e. soil sinking, was related to water content. These experimental data were compared with theoretical values calculated on the hypothesis of isotropic shrinkage of aggregates, and their continuous piling within the layer. The shrinkage of the aggregates was found to be isotropic. The sinking of the soil layers appeared to occur in two stages, which indicates changes in structural organization : the structural units move away at the beginning of drying, then they are brought nearer again in the second stage of drying.

Additional key words : Soil shrinkage, bulk density, soil structure, structural porosity.

\section{INTRODUCTION}

Dans les sols riches en argile gonflante, les variations d'humidité causent le retrait ou le gonflement des matériaux argileux et entraînent une modification de la densité qui est particulièrement sensible à l'échelle des agrégats.

Le retrait des agrégats résulte d'une diminution de leur porosité interne, c'est-à-dire d'un « transfert » de porosité de l'intérieur vers l'extérieur des agrégats. Il a pour conséquence une réorganisation géométrique qui se manifeste de 2 façons : la fissuration et l'affaissement.

(*) Adresse actuelle : I.N.R.A., Laboratoire de Science du Sol. Centre de recherches de Rennes, 65, rue de St-Brieuc, F 35042 Rennes Cedex.
La fissuration peut être considérée comme une création d'un espace poral : le retrait des agrégats se réalise au profit d'une porosité interagrégat; elle se traduit donc par une modification qualitative mais non quantitative de la porosité totale du sol.

L'affaissement, qui provient de l'effondrement des agrégats les uns sur les autres à la suite de leur retrait vertical, se traduit au contraire par une diminution de la porosité du sol, par réduction de son épaisseur.

Dans leur tentative de modélisation des phénomènes de retrait et de gonflement, certains auteurs, cherchant à évaluer la part respective de l'affaissement et de la fissuration, ont admis l'isotropie du retrait dans les 3 dimensions de l'espace (REEVE et al., 1980 ; HACK, 1984). Cette hypothèse est confirmée par divers travaux expérimentaux réalisés en laboratoire ou en conditions naturelles, notamment par WHITE (1962), 
JAMISON \& THOMPSON (1967), YAALON \& KALMAR (1972), BERNDT \& COUGHLAN (1977), YULE \& RITCHIE (1980), CABIDOCHE et al. (1986).

Cet article propose des méthodes de mesure du retrait vertical d'un sol argileux à 2 niveaux : sur des agrégats naturels soumis à l'évaporation au laboratoire, et sur des couches de sol desséchées in situ par une culture. Ces mesures de l'affaissement seront comparées aux valeurs calculées selon l'hypothèse d'un retrait isotrope ; à partir de cette comparaison, nous envisagerons les conséquences de l'affaissement sur l'organisation structurale du sol.

\section{MATÉRIEL ET MÉTHODE}

\section{A. Le sol}

Le sol étudié est situé aux Vignères, dans la plaine du Comtat Venaissin, à $20 \mathrm{~km}$ à l'Est d'Avignon. Il s'est développé sur des dépôts alluviaux récents, et présente environ $2 \mathrm{~m}$ de matériaux riches en argile, d'origine lacustre (plaine des Sorgues), reposant sur des galets mélangés à des lentilles sableuses et coquillières, d'origine fluviatile (Rhône-Durance-Calavon), où circule une nappe permanente. Pour les besoins de cette étude, le sol a été analysé sur une profondeur de $90 \mathrm{~cm}$, ce qui correspond à la profondeur d'enracinement de la prairie temporaire (fétuque élevée, âgée de 6 ans) qui en assure le dessèchement.

Le tableau 1 donne les caractéristiques granulométriques sur cette profondeur : la texture argileuse y est homogène. Les observations permettent de décrire une structure présentant une tendance cubique marquée de taille approximativement centimétrique jusqu'à $60 \mathrm{~cm}$, sensiblement plus grossière en-dessous.

\section{TABLEAU 1}

Caractéristiques granulométriques du sol étudié.

Textural characteristics of the studied soil.

\begin{tabular}{|c|c|c|c|c|c|c|}
\hline \multirow{2}{*}{ Profondeur } & \multicolumn{5}{|c|}{ Classes granulométrique $(\mu \mathrm{m}) \%$} & \multirow{2}{*}{$\begin{array}{c}\text { Calcaire } \\
\text { total } \\
\% 0\end{array}$} \\
\hline & $0-2$ & $2-20$ & $20-50$ & $50-200$ & $200-2000$ & \\
\hline $20 \mathrm{~cm}$ & 51,3 & 30,4 & 7,4 & 8,6 & 2,3 & 27,5 \\
\hline $40 \mathrm{~cm}$ & 51,5 & 29,6 & 7,3 & 9,1 & 2,5 & 28,2 \\
\hline $60 \mathrm{~cm}$ & 53,2 & 31,1 & 6,5 & 7,3 & 1,9 & 29,1 \\
\hline $80 \mathrm{~cm}$ & 54,9 & 32,6 & 5,4 & 5,6 & 1,5 & 29,5 \\
\hline $100 \mathrm{~cm}$ & 53,2 & 34,9 & 4,5 & 5,6 & 1,8 & 32,3 \\
\hline
\end{tabular}

Pour les besoins de cette étude, malgré l'absence d'horizons pédologiques nettement différenciés, il a été nécessaire de subdiviser le profil en couches dans lesquelles le gradient de teneur en eau pouvait être considéré comme négligeable : 4 couches de sol d'épaisseur $20 \mathrm{~cm}$ ont ainsi été distinguées entre 10 et $90 \mathrm{~cm}$; les $10 \mathrm{~cm}$ supérieurs, caractérisés par un mat racinaire dense, sont exclus de l'analyse. Ces couches seront dénommées A $(10-30 \mathrm{~cm}), \mathrm{B}(30-50 \mathrm{~cm}), \mathrm{C}(50-70 \mathrm{~cm})$ et $D(70-90 \mathrm{~cm})$.

L'importance de la fraction argileuse, et la présence au sein de cette fraction de minéraux à fort pouvoir de gonflement (montmorillonite) rendent nécessaire l'étude des courbes de retrait. Celles-ci ont été réalisées selon la méthode décrite par MONNIER et al. (1973), sur des petits agrégats naturels de 2-3 $\mathrm{mm}$. Les courbes, représentant les variations de volume des agrégats en fonction de leur humidité pondérale, peuvent être assimilées à des droites pour des teneurs en eau comprises entre $35 \%$ et $11 \%$ environ, c'est-à-dire dans tout le domaine d'humidité rencontré in situ: le tableau 2 propose les équations reliant la masse volumique sèche $\gamma_{\tau}$ des agrégats à leur teneur en eau pondérale $\mathrm{w}$, mesurée après séchage à $105^{\circ} \mathrm{C}$.

\section{TABLEAU 2}

Equations des droites de retrait des agrégats (pour $w>11 \%$ ). Equations of the shrinkage curves of the aggregates (for $w>11 \%$ ).

\begin{tabular}{rll}
\hline $20 \mathrm{~cm}$ & $1 / \gamma_{\tau}=0,881 \mathrm{w}+0,426$ & $\left(\mathrm{r}^{2}=0,989\right)$ \\
$40 \mathrm{~cm}$ & $1 / \gamma_{\tau}=0,987 \mathrm{w}+0,388$ & $\left(\mathrm{r}^{2}=0,991\right)$ \\
$60 \mathrm{~cm}$ & $1 / \gamma_{\tau}=0,918 \mathrm{w}+0,399$ & $\left(\mathrm{r}^{2}=0,992\right)$ \\
$80 \mathrm{~cm}$ & $1 / \gamma_{\tau}=0,898 \mathrm{w}+0,401$ & $\left(\mathrm{r}^{2}=0,985\right)$ \\
$100 \mathrm{~cm}$ & $1 / \gamma_{\tau}=0,986 \mathrm{w}+0,396$ & $\left(\mathrm{r}^{2}=0,968\right)$ \\
\hline
\end{tabular}

On peut par ailleurs remarquer que ces droites de retrait, dont la pente est voisine de 1 , sont peu différentes des droites de saturation, calculées à partir de la densité de solide du matériau (mesuree par la méthode du pycnometre à eau) : nous admettrons donc ici que les agrégats restent saturés dans tout le domaine d'étude. Notons toutefois que, compte tenu des incertitudes sur les mesures de densité, cette hypothèse de saturation des agrégats demanderait à être confirmée par des mesures effectuées sur une pâte saturée.

\section{B. Mesure du retrait vertical des agrégats}

La structure cubique particulièrement bien développée du sol étudié permet d'obtenir des agrégats de 5$10 \mathrm{~mm}$ à partir de mottes prélevées sur des parois de fosse à différentes profondeurs; leur orientation in situ est soigneusement repérée lors du prélèvement. Amenés à saturation, ces agrégats sont ensuite déposés sur le plateau d'une balance avec la même orientation, de façon à présenter à l'opérateur une face latérale.

Le dessèchement se fait par évaporation; tout au long du retrait, les agrégats sont photographiés et pesés. Des agrandissements photographiques permettront ensuite de mesurer avec précision le retrait vertical et le retrait latéral des agrégats, et de relier ces valeurs à leur humidité.

A la fin du dessèchement, la masse volumique des agrégats est mesurée par poussée d'Archimède dans le pétrole.

\section{Mesure du retrait vertical des couches de sol}

Les variations d'épaisseur des couches de sol sont mesurées à l'aide de 2 types de capteurs (fig. 1) :

- le capteur de type 1 , dérivé de celui décrit par SELIG \& REINIG (1982), est constitué d'un élément mobile placé à une profondeur déterminée (disque PVC de diamètre $20 \mathrm{~cm}$ ) surmonté d'un tube dépassant du sol ; ce disque et ce tube suivent les mouvements 


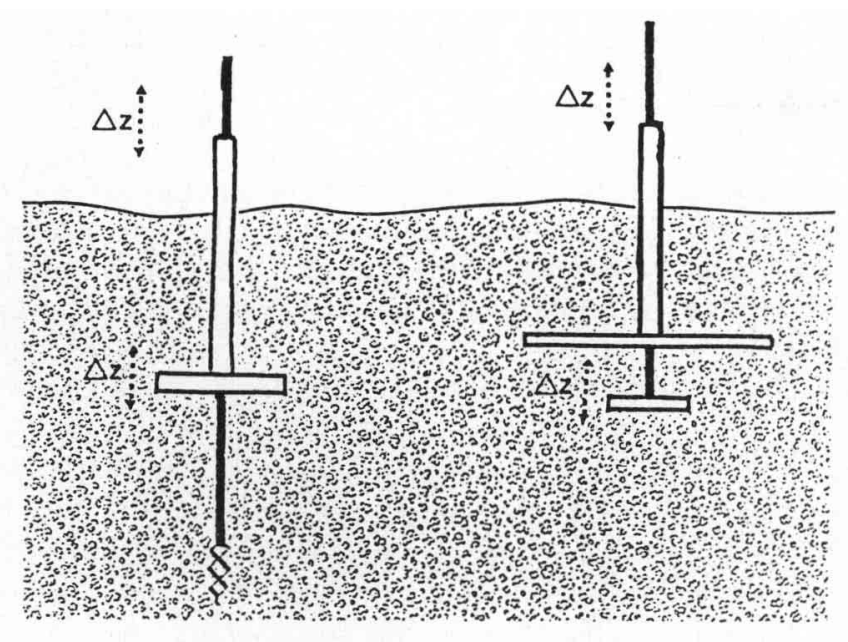

type i type II

Figure 1

Capteurs de déplacement vertical des couches de sol. Type I: mesure des variations de cote d'un niveau par rapport à un niveau de référence. Type II : mesure des variations d'épaisseur d'une couche. Vertical displacement transducers for soil layers. Type I : measurement of the variations of a level height in relation to a reference level. Type II : measurement of the variations of the thickness of a layer.

verticaux du sol en coulissant autour d'une tige ancrée dans un niveau de référence. On peut ainsi suivre depuis la surface les variations de cote d'un niveau donné. Les variations d'épaisseur d'une couche sont calculées par différence entre les variations du niveau supérieur et du niveau inférieur de cette couche. Sous prairie, 5 capteurs de ce type ont été placés à chacune des 5 profondeurs suivantes: $10 \mathrm{~cm}, 30 \mathrm{~cm}, 50 \mathrm{~cm}$, $70 \mathrm{~cm}$ et $90 \mathrm{~cm}$, qui délimitent les 4 couches de sol ;

- le capteur de type II est constitué de 2 éléments mobiles : la partie inférieure est un disque PVC de diamètre $20 \mathrm{~cm}$, la partie supérieure un rectangle de $15 \mathrm{~cm} \times 70 \mathrm{~cm}$. Comme précédemment, ces éléments sont solidaires de tubes qui coulissent l'un dans l'autre et dépassent du sol. Les variations d'épaisseur d'une couche de sol peuvent ainsi être lues directement depuis la surface. Cinq capteurs de ce type ont été placés pour chacune des 4 couches étudiées.

Les capteurs sont disposés au fond d'une fosse creusée à la profondeur voulue, rebouchée après mise en place du capteur. Il s'ensuit une forte perturbation du matériau situé au-dessus de la couche dont on mesure les variations de cote : cette perturbation se fait sentir dans l'année qui suit la mise en place. Une grande variabilité de la réponse des capteurs a pu être imputée aux conditions de circulation d'eau particulières au sein de ce matériau remanié. Dès la seconde année par contre, cette variabilité devient négligeable : on considèrera que les risques d'artefact liés à la mise en place sont alors nuls.

Sur toute une saison de végétation (du 15 mars au 30 août), les cotes des capteurs ont été relevées 116 fois, soit en moyenne 3 jours sur 4 . Cette période a été entrecoupée de 2 épisodes pluvieux importants qui ont permis une réhumectation partielle, ou même totale pour les couches supérieures ; les résultats portent donc sur 3 phases de dessèchement.
L'humidité est déterminée après séchage à $105^{\circ} \mathrm{C}$ d'échantillons prélevés à la tarière au niveau médian de chaque couche étudiée $(20,40,60$ et $80 \mathrm{~cm})$. Lors de chaque relevé de cote, 10 profils hydriques sont réalisés. A ces mesures gravimétriques s'ajoutent des mesures du potentiel hydrique obtenues par la méthode décrite par BRUCKLER (1984) au moyen de micropsychromètres placés au voisinage immédiat des capteurs de type II : ces mesures ne mettent pas en évidence de différence d'humidité significative sous les capteurs et hors capteurs.

Par ailleurs, afin de pouvoir convertir l'épaisseur en masse volumique, des mesures densimétriques ont été effectuées en hiver, alors que le sol était saturé : sur chaque couche de sol, on a prélevé 10 cylindres de volume connu (hauteur $7,2 \mathrm{~cm}$, diamètre $15 \mathrm{~cm}$ ), ce volume étant considéré, pour cette teneur en eau, comme représentatif de la couche.

\section{RÉSULTATS}

\section{A. Affaissement des agrégats}

Seuls seront présentés ici les résultats obtenus sur des agrégats prélevés à $40 \mathrm{~cm}$ de profondeur ; les résultats obtenus à d'autres profondeurs sont identiques.

La figure 2 présente le retrait vertical et le retrait

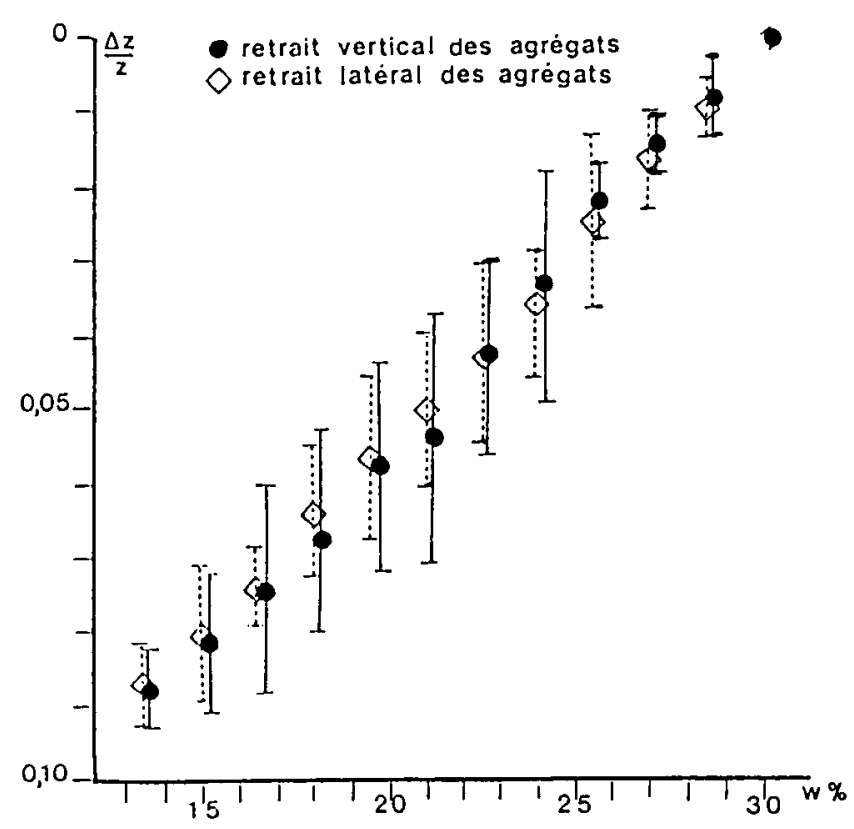

Figure 2

Variations de la hauteur et de la largeur des agrégats $\Delta z / z$ avec l'humidité pondérale w. Moyennes et écarts-types calculés sur 6 agrégats de la couche $B(30-50 \mathrm{~cm})$.

Variation in the height and width of the aggregates $\Delta z / z$ with gravimetric water content $w$. Means and standard deviations calculated on 6 aggregates from the $B$ layer $(30-50 \mathrm{~cm})$.

latéral, mesurés sur 6 agrégats, les valeurs étant ramenées aux valeurs initiales; les résultats sont exprimés par la moyenne et l'écart-type du retrait en fonction de la teneur en eau pondéral $w$.

Ce graphe fait apparaître une importante dispersion des résultats tout au long du dessèchement, ne se réduisant que pour des teneurs en eau inférieures à 
15 p. 100. Cette forte hétérogénéité peut avoir 2 causes :

- d'une part les mesures de hauteur et de largeur des agrégats sur agrandissements photographiques ne sont pas assez précises pour mettre en évidence de petites variations ; remédier à cette imprécision nécessiterait soit de procéder à de plus forts agrandissements (au risque toutefois d'une perte de netteté de l'image), soit de prendre en compte un plus grand nombre d'agrégats ;

- d'autre part les différents agrégats que l'on mesure sur une image peuvent présenter une hétérogénéité hydrique, non mesurable sur une population d'agrégats dont on ne connaît que l'humidité moyenne. Cette hétérogénéité hydrique est particulièrement élevée dans la gamme de teneur en eau où la dispersion des résultats est la plus grande, entre 24 p. 100 et $20 \mathrm{p} .100$ : les agrégats situés sur le pourtour du plateau de la balance se dessèchant plus rapidement que ceux situés au centre, on ne peut pas maîtriser la variabilité de l'humidité en un instant donné.

Ces imprécisions constituent un inconvénient majeur pour l'utilisation de cette méthode en dehors de l'objectif que nous nous sommes fixés ici : elle ne saurait, en tout état de cause, se substituer à la méthode classique (mesure de la poussée d'Archimède dans le pétrole) lorsqu'on ne cherche pas à distinguer le retrait vertical du retrait latéral.

Malgré ces réserves, cette méthode photographique nous permet de tirer les conclusions suivantes :

- lorsque la teneur en eau pondérale passe de 30 p. 100 à 13 p. 100, la taille des agrégats est réduite en moyenne de 9 p. 100 ; cette réduction apparaît, en première approximation, linéaire tout au long du dessèchement ;

- cette réduction de taille est identique dans les 3 dimensions de l'espace : les résultats ne permettent pas de mettre en évidence de différence significative entre les retraits vertical et latéral.

\section{B. Affaissement des couches de sol}

L'épaisseur d'une couche est mesurée soit par différence entre 2 capteurs de type I, soit directement sur un capteur de type II. Disposant de 5 capteurs de type I à chaque profondeur et de 5 capteurs de type II pour chaque couche, on peut donc calculer l'épaisseur d'une couche de $\mathbf{3 0}$ façons différentes. L'épaisseur moyenne d'une couche en un instant donné, calculée sur ces 30 valeurs, ne sera considérée comme significative que lorsque leur écart-type sera inférieur à $0,25 \mathrm{~mm}$. Sur les 116 relevés effectués durant la période d'étude, seuls 2 à 5 relevés (selon les couches) ont dû être rejetés pour cette raison; notons que les relevés présentant une trop forte dispersion correspondent tous à un regonflement du sol, consécutif à une réhumectation : le gonflement des couches semble s'opérer de façon plus hétérogène que leur affaissement.

Ces valeurs moyennes de l'affaissement des couches sont associées à leur humidité moyenne, calculée à partir des 10 profils hydriques réalisés lors de chaque relevé. Compte tenu de la très grande homogénéité hydrique au sein de la parcelle d'étude, ces valeurs d'humidité moyenne ont pu être considérées comme significatives durant toute la phase de dessèchement : ici encore les rares valeurs présentant une forte dispersion sont celles obtenues en phase de réhumectation.

L'homogénéité des variables mesurées au cours du dessèchement nous a donc permis de retenir, selon les couches, 111 à 114 couples teneur en eau-épaisseur de la couche : les variations d'épaisseur des couches A, B, $\mathrm{C}$ et $\mathrm{D}$ avec l'humidité pondérale w sont données sur la figure 3 .

Les résultats font apparaître les points suivants :

- les nuages de points sont assez étroits, ce qui révèle une bonne répétabilité des résultats entre les différentes phases de dessèchement ;

- l'allure des courbes d'affaissement au cours du dessèchement varie selon les couches : tandis que la variation d'épaisseur apparaît approximativement linéaire pour la couche la plus profonde, elle présente pour les autres couches un aspect nettement incurvé, avec un affaissement très peu marqué au début du dessèchement, et s'accentuant notablement en-dessous de 25 p. 100 pour devenir de plus en plus rapide ;

- l'affaissement des couches, sur toute la période de dessèchement, est d'autant plus faible que la couche est plus profonde : il passe de $1,6 \mathrm{~cm}$ pour la couche la plus superficielle (A) à $1 \mathrm{~cm}$ pour la couche la plus profonde (D), représentant ainsi 5 à 8 p. 100 de l'épaisseur initiale des couches $(20 \mathrm{~cm})$. Ceci peut être imputé au fait que le dessèchement est plus poussé en surface qu'en profondeur.

\section{DISCUSSION}

Au vu de nos résultats, la relation entre le retrait vertical et la teneur en eau apparaît d'ores et déjà complexe : elle est approximativement linéaire pour les agrégats et pour la couche profonde, nettement curviligne pour les couches supérieures.

Cependant la lourdeur des dispositifs expérimentaux montre tout l'intérêt qu'il y aurait à expliciter cette relation affaissement-humidité : c'est ainsi que, comme nous l'avons signalé plus haut, de nombreux auteurs ont admis que le retrait était isotrope. Cette notion d'isotropie, établie en premier lieu sur des échantillons de petite taille, a ensuite été étendue à l'échelle d'une couche de sol ou d'un profil.

Si nous voulons tester l'hypothèse d'isotropie du retrait sur les agrégats et sur les couches de sol, nous devons établir la relation liant l'affaissement à l'humidité lorsque le retrait est isotrope, puis comparer cette relation théorique à nos valeurs expériementales.

\section{A. L'équation du retrait isotrope des agrégats}

Le retrait d'un agrégat sera dit isotrope s'il est identique dans les 2 dimensions horizontales $\mathrm{x}$ et $\mathrm{y}$ et dans la dimension verticale $\mathrm{z}$ :

$$
\frac{\Delta x}{x}=\frac{\Delta y}{y}=\frac{\Delta z}{z} .
$$

L'hypothèse $d$ 'isotropie du retrait ne privilégiant aucune direction, le volume $\mathrm{V}$ d'un agrégat est, à tout 

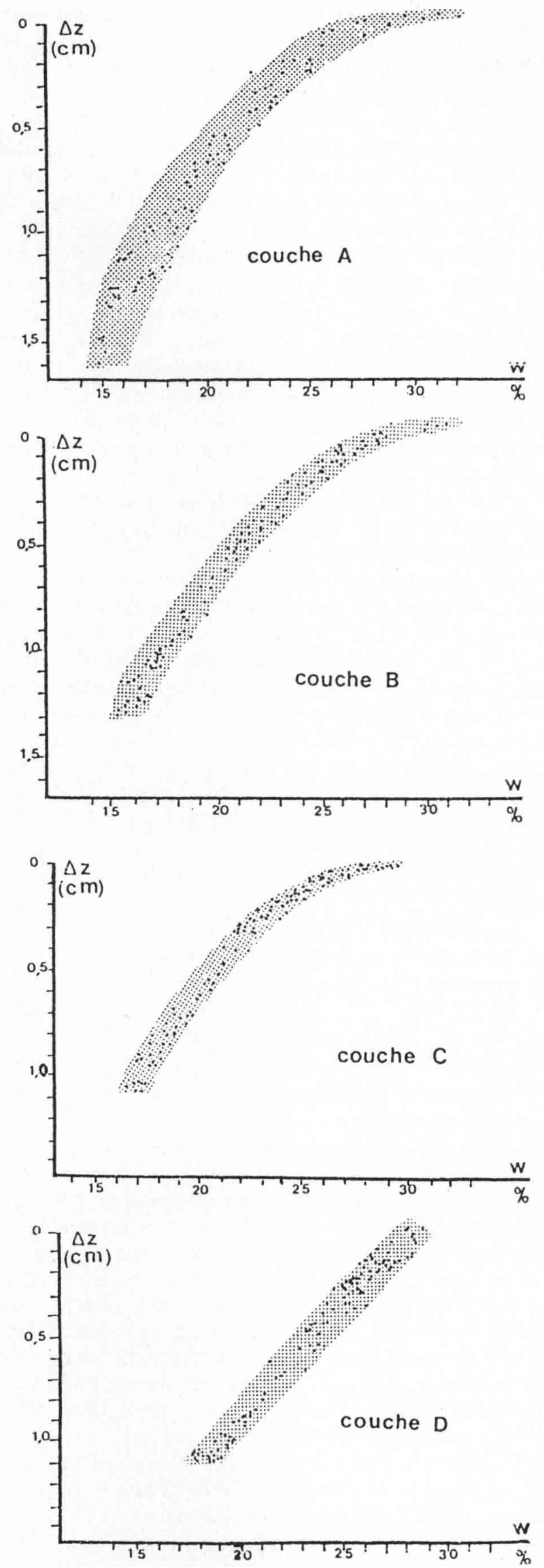

Figure 3

Variations d'épaisseur des couches de sol avec l'humidité pondérale w. Couches: $A(10-30 \mathrm{~cm}), B(30-50 \mathrm{~cm}), C(50-70 \mathrm{~cm})$, $D(70-90 \mathrm{~cm})$.

Variation in thickness of the soil layers with gravimetric water content w. Layers : $A(10-30 \mathrm{~cm}), B(30-50 \mathrm{~cm}), C(50-70 \mathrm{~cm}), D(70$ $90 \mathrm{~cm})$. instant, proportionnel au cube de son épaisseur z :

$$
\frac{\mathrm{V}}{\mathrm{V}_{0}}=\frac{\mathrm{z}^{3}}{\mathrm{z}_{0}^{3}}
$$

où $\mathrm{V}_{0}$ et $\mathrm{z}_{0}$ correspondent à un état initial donné.

$\mathrm{Si} \gamma_{\tau}$ est la masse volumique sèche d'un agrégat de volume $\mathrm{V}$ et d'épaisseur $\mathrm{z}$, on a donc :

$$
\frac{\mathrm{z}^{3}}{\mathrm{z}_{0}^{3}}=\frac{\gamma_{\tau 0}}{\gamma_{\tau}}
$$

Or nous avons vu précédemment que, dans tout le domaine des teneurs en eau rencontrées ( $w$ compris entre 30 p. 100 et 13 p. 100), la courbe de retrait des agrégats était très proche de la droite de saturation, et que leur masse volumique $\gamma_{\tau}$ était liée à leur humidité pondérale w par l'équation :

$$
1 / \gamma_{\tau}=a w+b .
$$

L'hypothèse d'isotropie du retrait d'un agrégat peut donc s'écrire :

$$
\frac{z^{3}}{z_{0}^{3}}=\gamma_{\pi 0}(a w+b)
$$

et l'affaissement « isotrope " prend la forme :

$$
\frac{\Delta \mathrm{z}}{\mathrm{z}_{0}}=\frac{\mathrm{z}_{0}-\mathrm{z}}{\mathrm{z}_{0}}=1-\sqrt[3]{\gamma_{\mathrm{r} 0}(\mathrm{aw}+\mathrm{b})} .
$$

Dans la gamme d'humidité qui nous intéresse, cette relation exponentielle entre $\Delta z$ et $w$ est peu différente d'une relation linéaire ; la courbe du retrait isotrope peut alors être assimilée à une droite.

\section{B. Isotropie du retrait des agrégats}

Les mesures n'ont pas permis de mettre en évidence de différence significative entre le retrait vertical et le retrait latéral des agrégats (fig. 2) : ce résultat nous avait incité à admettre l'isotropie de leur retrait.

Cette isotropie est confirmée par le calcul : la courbe théorique du retrait isotrope, obtenue en appliquant l'équation précédemment établie à la couche de sol concernée $(a=0,987 ; b=0,388)$ et à ses conditions initiales $\left(w_{0}=30\right.$ p. $\left.100 ; \gamma_{T 0}=1,468\right)$ passe précisément par les points expérimentaux.

Le dessèchement des agrégats provoque donc une diminution de leur volume sans modifier leur géométrie. Cette absence de déformation des agrégats, mise en évidence à un niveau macroscopique, ne s'oppose toutefois pas à ce qu'il y ait déformation des édifices à des échelles plus fines, c'est-à-dire réorganisation des porosités intercristallites et interdomaines (TESSIER, 1984).

\section{Extension au retrait des couches de sol}

Les mesures effectuées en période hivernale ont montré que, lorsque le sol est saturé, la porosité interagrégat est négligeable : la masse volumique des couches de sol n'est pas significativement différente de celle des agrégats. 
Au cours du dessèchement, alors que les agrégats se rétractent tout en conservant leur forme, on peut s'interroger sur la persistance de leur empilement au sein d'une couche. Si cet empilement reste continu, l'affaissement de la couche est proportionnel à celui des agrégats qui la composent ; il peut donc être calculé à l'aide de l'équation du retrait isotrope des agrégats.

Ce calcul a été effectué sur nos 4 couches de sol, à partir de leurs conditions initiales. La relation théorique, quasiment linéaire comme on l'a signalé plus haut, est ensuite comparée aux valeurs expérimentales ; c'est pour les couches $\mathrm{A}, \mathrm{B}$ et $\mathrm{C}$ que l'on observe les différences les plus importantes : on a vu que ces couches se caractérisaient par un affaissement très faible au début du dessèchement, pour devenir de plus en plus rapide. Si l'on traduit ces courbes d'affaissement en courbes de retrait, on peut rapprocher ce comportement de celui observé par BRUAND (1985), où les échantillons de taille « micrométrique " d'un sol argileux présentent un retrait linéaire (tout comme nos agrégats), tandis que les échantillons « centimétriques $»$ s'en écartent nettement (tout comme nos couches de sol).

Pour mieux mettre en évidence l'écart entre l'affaissement des couches de sol et celui des agrégats qui les composent, nous avons représenté (fig. 4) l'évolution, en fonction de l'humidité pondérale $w$, de la différence entre les valeurs calculées à partir de l'équation d'isotrophie, $(\Delta z)_{c}$, et les valeurs mesurées $(\Delta z)_{m}$.

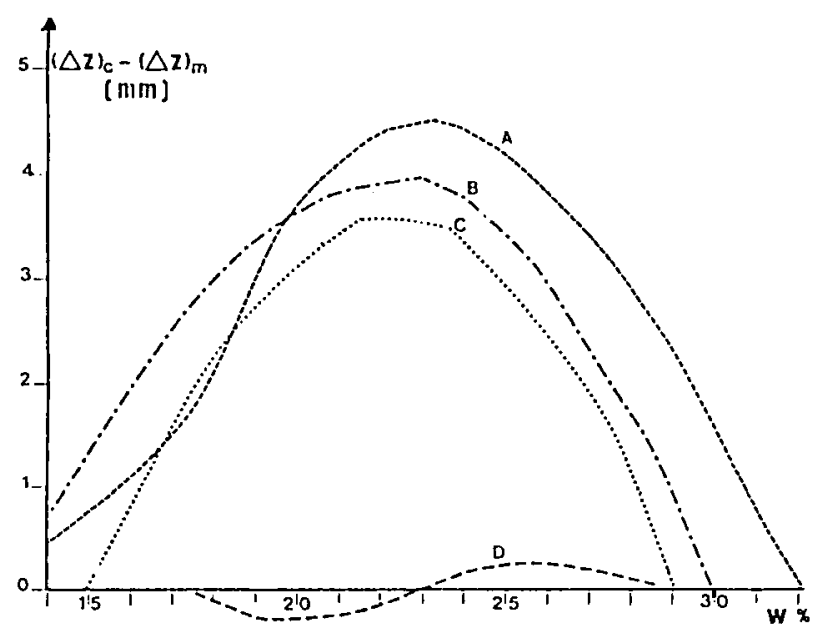

Figure 4

Variations de l'écart entre l'affaissement calculé $(\Delta z)_{c}$ et l'affaissement mesuré $(\Delta z)_{m}$ avec l'humidité pondérale $w$. Couches : A (10$30 \mathrm{~cm}), B(30-50 \mathrm{~cm}), C(50-70 \mathrm{~cm}), D(70-90 \mathrm{~cm})$.

Variation in the difference between the calculated sinking $(\Delta z)_{c}$ and the measured sinking $(\Delta z)_{m}$ with gravimetric water content $w$. Layers : $A(10-30 \mathrm{~cm}), B(30-50 \mathrm{~cm}), C(50-70 \mathrm{~cm}), D(70-90 \mathrm{~cm})$.

Pour la couche la plus profonde, cet écart reste très faible quelle que soit l'humidité : on peut donc admettre que, tout au long du dessèchement, les agrégats de cette couche conservent l'empilement continu qu'ils avaient à saturation : leur comportement isotrope se répercute à l'échelle de la couche.

Pour les 3 couches supérieures, cet écart présente une variation caractéristique au cours du dessèchement : dans une première phase (jusqu'à une humidité de 22-23 p. 100 environ), l'écart entre l'affaissement réel de la couche et celui de ses agrégats s'accroît ; endessous de cette humidité, cet écart diminue et tend à s'annuler en fin de dessèchement.

Si l'on veut analyser cette variation d'affaissement pour l'inclure dans un schéma d'évolution structurale du sol, il nous faut intégrer le comportement de ce sol à la fissuration (HALLAIRE, 1984); cette analyse en terme de bilan poral fera l'objet d'une étude ultérieure, où seront discutés notamment les relations entre affaissement et fissuration, et la part respective de chacun de ces processus dans le retrait total du sol.

En nous limitant pour l'instant à la seule composante verticale du retrait, nous constatons que l'affaissement des 3 couches supérieures du sol est moindre que celui de leurs agrégats. Compte tenu de l'isotropie du retrait de ces agrégats, ce "retard à l'affaissement " nous amène à envisager l'ouverture, au cours du dessèchement, d'une porosité qui résulterait d'une superposition imparfaite des agrégats les uns au-dessus des autres. Cette composante horizontale de la porosité structurale augmente jusqu'au taux d'humidité de 23 p. 100 où elle atteint son maximum ; puis elle diminue jusqu'à la fin du dessèchement où les agrégats retrouvent leur empilement continu. La différence $(\Delta z)_{c}-(\Delta z)_{m}$ représentée sur la figure 4 correspondrait ainsi à la somme des vides unitaires résultant de cette mauvaise superposition. On remarque que cet écart s'amenuise en profondeur: il atteint $4,5 \mathrm{~mm}$ en $\mathrm{A}, 4 \mathrm{~mm}$ en $\mathrm{B}$, et $3,5 \mathrm{~mm}$ en $\mathrm{C}$; cette diminution peut s'expliquer par l'effet gravitaire que la colonne de sol susjacente oppose à cette ouverture, et qui est bien évidemment plus important en profondeur qu'en surface.

Les méthodes utilisées ici nous ont permis de mettre en évidence cette fraction porale ; mais elles ne nous donnent aucun renseignement sur sa morphologie. Aussi serait-il risqué de l'assimiler à une "fissuration horizontale ", qui n'aurait aucune signification structurale.

L'existence de cette composante horizontale de la porosité laisse toutefois supposer qu'elle est constituée de vides suffisamment fins pour que subsistent entre les éléments structuraux des points de contact empêchant leur superposition parfaite, et s'opposant ainsi à l'effondrement total de la couche. L'ouverture de cet espace poral au cours de la première phase de dessèchement pourrait résulter d'une hétérogénéité hydrique entre les différents agrégats d'une même couche, contrariant ainsi leur empilement parfait ; une homogénéisation progressive de l'humidité en-dessous de 22 p. 100 permettrait ensuite la reconstitution de l'empilement initial des agrégats, refermant alors les vides précédemment ouverts.

Il n'est pas possible dans l'état actuel d'expliquer pourquoi seule la couche profonde présente un retrait isotrope : les caractéristiques physiques et chimiques ne suffisent pas à rendre compte du changement brutal de comportement entre les couches $\mathrm{A}, \mathrm{B}$ et $\mathrm{C}$ d'une part, D d'autre part. Pour comprendre le caractère anisotrope des couches supérieures, il faudrait sans doute relier le potentiel hydrique correspondant au seuil de 23 p. 100 au comportement rhéologique du matériau : FAURE (1978) et GRIMALDI (1981) ont montré l'incidence des contraintes hydriques sur la plasti- 
cité des matériaux argileux, GUÉRIF (1982) a mis en évidence des valeurs seuils de "viscoélasticité 》 pour des massifs d'agrégats.

\section{CONCLUSION}

Il n'est pas possible de négliger dans une analyse de la porosité l'affaissement du sol, qui peut représenter plus de 5 p. 100 de la hauteur d'un sol argileux, et atteint même dans notre cas 8 p. 100 pour la couche supérieure. La vérification expérimentale de l'isotropie du retrait sur certains types de sol a pu amener de nombreux auteurs à adopter des modèles simples : l'hypothèse d'isotropie permet de calculer aisément l'affaissement du sol à partir des variations d'humidité, sans plus nécessiter un dispositif expérimental lourd à mettre en œuvre.

Nos résultats s'opposent à la généralisation de cette hypothèse : l'analyse du sol étudié ici a permis de constater que l'isotropie du retrait des agrégats ne suffisait pas dans tous les cas à assurer un comportement isotrope au niveau d'une couche de sol. Le problème se complique encore lorsque les agrégats eux-mêmes présentent un caractère anisotrope : TOWNER (1986) a ainsi pu mettre en évidence l'incidence d'une contrainte mécanique sur l'anisotropie du retrait à l'échelle de petits échantillons. En tout état de cause, ces résultats montrent le danger de généraliser le modèle de retrait isotrope tant que l'on n'a pas vérifié, d'une part l'isotropie du retrait des éléments structu- raux, d'autre part la persistance, tout au long du dessèchement, de la superposition parfaite de ces éléments structuraux : un tel calcul de l'affaissement, sans justification des hypothèses, ne paraît pas pouvoir se substituer à des mesures in situ.

Les problèmes soulevés par une telle anisotropie sont de 3 ordres :

- une meilleure compréhension du mécanisme mis en œuvre nous aiderait à mieux cerner les types de sol concernés par ce phénomène, et à expliquer la différence de comportement de la couche profonde. L'étude des propriétés rhéologiques du matériau, notamment autour de la valeur seuil d'humidité de 23 p. 100, devrait contribuer à cette analyse ;

- les méthodes utilisées mettent en évidence la création, au cours du dessèchement, d'une composante de la porosité structurale qualifiée d'horizontale, résultant d'une mauvaise continuité verticale des agrégats. Si l'on peut qualifier globalement cette fraction porale, il reste à en proposer une description morphologique : cet aspect sera repris dans une étude ultérieure, où l'analyse intégrera affaissement et fissuration ;

- les conséquences d'une telle évolution structurale sur le comportement du sol apparaissent d'ores et déjà importantes : l'écartement puis le rapprochement des éléments structuraux sont des données indispensables à la prévision des transferts liquides ou gazeux, des possibilités de pénétration racinaire, et de la susceptibilité au tassement.

Reçu le 12 avril 1986. Accepté le 6 mai 1987.

\section{RÉFÉRENCES BIBLIOGRAPHIQUES}

Berndt R. D., Coughlan K. J., 1977. The nature of change in bulk density with water content in a cracking clay. Aust. J. Soil Res., 15 (1), 27-37.

Bruand A., 1985. Contribution à l'étude de la dynamique de l'organisation de matériaux gonflants. Thèse de Spéc. Paris VII, 225 p.

Bruckler L., 1984. Utilisation des micropsychromètres pour la mesure du potentiel hydrique du sol en laboratoire et in situ. Agronomie, 4 (2), 171-182.

Cabidoche Y. M., Jaillard B., Ney B., 1986. Dynamique de l'eau dans les vertisols sur calcaires récifaux, pp. 449-478. In : Sol et Eau, Actes du Séminaire de La Havane, 8-20 avril 1985, ORSTOM, Paris, $767 \mathrm{p}$.

Faure A., 1978. Comportement des sols au compactage : rôle de l'argile et conséquences sur l'arrangement des grains. Thèse Doct. ès Sci. Grenoble, $179 \mathrm{p}$.

Grimaldi M., 1981. Contribution à l'étude du tassement des sols : Evolution de la structure d'un matériau limoneux soumis à des contraintes mécaniques et hydriques. Thèse ENSA Rennes, $220 \mathrm{p}$.

Guérif J., 1982. Compactage d'un massif d'agrégats : effet de la teneur en eau et de la pression appliquée. Agronomie, 2 (3), 287-294.

Hack H. R. B., 1984. Calculation of the field volumetric water content of cracking clay soils from measurements of gravimetric water content and bulk density. J. Soil Sci., 35 (2), 299-315.

Hallaire V., 1984. Evolution of crack networks during shrinkage of a clay soil, pp. 49-54, In : Proc. of the ISSS Symp. on Water and Solute Movement in Heavy Clay Soils, ILRI Publication 37, Wageningen, $363 \mathrm{p}$.
Jamison V. C., Thompson G. A., 1967. Layer thickness changes in a clay-rich soil in relation to soil water content changes. Soil Sci. Soc. Am. Proc., 31, 441-444.

Monnier G., Stengel P., Fiès J. C., 1973. Une méthode de mesure de la densité apparente de petits agglomérats terreux. Application à l'analyse des systèmes de porosité du sol. Ann. agron., 24 (5), 533-545.

Reeve M. J., Hall D. G. M., Bullock P., 1980. The effect of soil composition and environmental factors on the shrinkage of some clayey British soils. J. Soil Sci, 31 (3), 429-442.

Selig E. T., Reinig I. G., 1982. Vertical soil extensometer. Geotechn. Test. J., 5 (3/4), 76-84.

Tessier D., 1984. Etude expérimentale de l'organisation des minéraux argileux. Thèse Doct. ès Sci. Paris VII, I.N.R.A., 361 p.

Towner G. D., 1986. Anisotropic shrinkage of clay cores, and the interpretation of field observations of vertical soil movement. J. Soil Sci., 37 (3), 363-371.

White E. M., 1962. Volume change in some clay soils. Soil Sci., 94 (3), 168-172.

Yaalon D. H., Kalmar D., 1972. Vertical movement in undisturbed soil : continuous measurement of swelling and shrinkage with a sensitive apparatus. Geoderma, 8 (4), 231-240.

Yule D. F., Ritchie J. T., 1980. Soil shrinkage relationships in Texas Vertisols. I. Small cores. II. Large cores. Soil Sci. Soc. Am. J., 44 (6), 1285-1291 et 192-1295. 